\title{
Pathways Between Internalized Stigma and Outcomes Related to Recovery in Schizophrenia Spectrum Disorders
}

\author{
Philip T. Yanos, Ph.D. ${ }^{1}$, David Roe, Ph.D. ${ }^{2}$, Keith Markus, Ph.D. ${ }^{1}$, and Paul H. Lysaker, Ph.D. \\ 3 \\ 1 Department of Psychology, John Jay College of Criminal Justice-City University of New York, 445 W. 59th \\ St., New York, NY 10019
}

2 Department of Community Mental Health, University of Haifa, Haifa, Israel

3 Roudebush Veterans Affairs Medical Center Day Hospital, Indianapolis, Indiana

\section{Abstract}

Objective-The mechanisms by which internalized stigma affects outcomes related to recovery among people with severe mental illness have yet to be explicitly studied. This study empirically evaluated a model for how internalized stigma affects important outcomes related to recovery.

Methods-A total of 102 persons with schizophrenia spectrum disorders completed measures of internalized stigma, awareness of mental illness, psychiatric symptoms, self-esteem, hopefulness, and coping. Path analyses tested a predicted model and an alternative model for the relationships between the variables.

Results-Results from model 1 supported the view that internalized stigma increases avoidant coping, active social avoidance, and depressive symptoms and that these relationships are mediated by the impact of internalized stigma on hope and self-esteem. Results from model 2 replicated significant relationships from model 1 but also supported the hypothesis that positive symptoms may influence hope and self-esteem.

Conclusions-Findings from two models supported the hypothesis that internalized stigma affects hope and self-esteem, leading to negative outcomes related to recovery. It is recommended that interventions be developed and tested to address the important effects of internalized stigma on recovery.

Research has increasingly revealed that outcomes for persons with severe mental illness encompass a wide range of possibilities and challenges. As noted by several authors (1-3), what is referred to as recovery involves nonlinear changes across several semi-independent domains. Recovery can be manifested in more externally evident and objectively measured changes such as symptoms, frequency of socialization, and employment (2), as well in terms of more internally experienced and subjectively measured domains such as perceived quality of life and sense of purpose in life (3). The personal experience of mental illness and the potential transformation of identity that often accompanies this experience has been identified as a potential facilitator or inhibitor of recovery among people with severe mental illness (4), and there is evidence that transforming identity is an important part of the process of improving both subjective and objective outcomes in this population $(5,6)$.

e-mail: pyanos@jjay.cuny.edu.

The authors report no competing interests. 
A type of identity transformation that may affect many people with severe mental illness is the internalization of stereotypic or stigmatizing views (7-11). The state in which a person with severe mental illness loses previously held or hoped for identities (self as student, self as worker, self as parent, and so on) and adopts stigmatizing views (self as dangerous, self as incompetent, and so on) is typically referred to as "internalized stigma." As an illustration, a person with a college degree and prior aspirations to become a math teacher might conclude that he or she could never achieve this goal if he or she believes that the diagnosis of mental illness means that one is unpredictable and not to be trusted. Internalized stigma has been found to be relatively common among people with severe mental illness $(7,10)$ and to be significantly negatively related to hopefulness, self-esteem, and social functioning (7-11). Nevertheless, the way in which internalized stigma affects outcomes related to recovery and its relative importance in the context of other variables that may significantly affect recovery (such as symptom severity) has yet to be explicitly studied.

On the basis of prior empirical work and theory, we proposed a model for how internalized stigma affects recovery-related outcomes among people with severe mental illness (Yanos PT, Roe D, Lysaker PH, unpublished manuscript, 2008). The model is presented in Figure 1. As the model indicates, we hypothesized that an individual's awareness of having a psychiatric problem interacts with the meanings that the person attributes to the problem. This interaction then directly affects hopefulness and self-esteem. Internalization of stigma can lead to a sense of hopelessness about the possibility of recovering and other negative self-evaluations. Hopelessness and negative evaluations, in turn, lead to low engagement in rehabilitation treatment and other services and a tendency to use avoidant coping strategies to deal with symptoms and stressors. Simultaneously, there is increased social isolation because of social anxiety, and risk of suicide is increased. Avoidant coping and lack of engagement in rehabilitation and treatment, in turn, lead to poorer vocational outcomes, which are further compounded by lack of social support resulting from social isolation. Finally, we hypothesized that the types of coping strategies used, social interactions, and vocational functioning all affect the severity of psychotic symptoms. We should make clear that we do not hypothesize that psychotic symptoms are caused by these factors, but rather that psychotic symptoms can become more or less severe and disabling, depending on the degree to which individuals remain socially isolated, have or lack the structure of employment, and continue to use avoidant coping strategies. There is empirical evidence that involvement in competitive work, for example, affects severity of psychotic symptoms (12).

Although different aspects of the associations we predict in the model have been tested by various investigators (Yanos and colleagues, unpublished paper, 2008), the purpose of this study was to conduct a more comprehensive empirical evaluation of the model. To achieve this goal and to provide a partial test of the model, we conducted a path analysis of secondary data with a sample of persons diagnosed as having schizophrenia spectrum disorders. All participants were unemployed, so vocational status was equivalent in the sample. Path analysis provides a means of testing complex causal models by estimating several regression equations at once. The fit of the model is evaluated statistically in terms of the fidelity with which the model reproduces the observed covariation between the variables. In addition to testing our proposed model, we tested a recent alternative model based on evidence provided by Fialko and colleagues (13), which suggests that psychotic symptoms such as hallucinations and delusions might be a cause, rather than a consequence, of diminished hope and self-esteem.

\section{Methods}

\section{Participants}

A total of 102 persons ( 87 men and 15 women) had diagnoses of schizophrenia spectrum disorders (68 with schizophrenia and 34 with schizoaffective disorder), confirmed with the 
Structured Clinical Interview for DSM-IV (14). They were recruited from a comprehensive day hospital at a Veterans Affairs medical center $(\mathrm{N}=70)$ and local community mental health center $(\mathrm{N}=32)$ for a study of the effects of cognitive-behavioral therapy on vocational rehabilitation. Institutional review board approval was received for the study, and all participants provided informed consent before participating in the research. All participants were receiving ongoing outpatient treatment and were in a postacute or stable phase of their disorder, defined as no hospitalizations or changes in medication or housing in the past month. Participants with a history of mental retardation, documented in a chart review, were excluded from the study. Participants had a mean $\pm \mathrm{SD}$ age of $46.23 \pm 9.62$ and a mean education of 12.55 \pm 2.24 years. They had $7.06 \pm 8.65$ lifetime hospitalizations, with the first occurring on average at the age of 27.12 \pm 9.99 . Forty-two (41\%) participants were Caucasian, $59(58 \%)$ were African American, and one (1\%) was Latino. The study was conducted between September 2004 and September 2007.

\section{Measures}

The Positive and Negative Syndrome Scale (PANSS) is a 30-item rating scale completed by clinically trained research staff at the conclusion of chart review and a semistructured interview (15). For our analysis we used single items (social avoidance and depression) and a factoranalytically derived component (positive symptoms, which includes symptoms such as hallucinations and delusions) (16). Single items were used where multiple items were not available for representing the constructs of interest (depressive symptoms and social avoidance).

The Scale for Assessing Unawareness of Mental Disorder (SUMD) is a three-item rating scale completed after a semistructured interview (17). For the purposes of this study, we used the total score, which is the sum of the three central items of the SUMD: awareness of mental disorder, awareness of the consequences of mental disorder, and awareness of the effects of medication. Each of these items is rated on a 5-point scale that ranges from 1, complete awareness, to 5, severe unawareness; thus higher scores indicate lower awareness. Assessment of interrater reliability for raters in this study was in the good to excellent range (intra-class $\mathrm{r}=.90)$.

The Internalized Stigma of Mental Illness Scale (ISMIS) is a 29-item paper-and-pencil questionnaire designed to assess subjective experience of stigma (18). Items from two subscales-alienation, which reflects feeling devalued as a member of society, and stereotype endorsement, which reflects agreement with negative stereotypes of mental illness-were summed to provide one measure of internalized stigma. All scale scores were calculated as averages, with higher scores suggesting graver experiences of stigma. The instrument in this study was presented to persons in its printed form, and research assistants were available to assist if participants were confused about the meaning of any item. Evidence of acceptable internal consistency ( $\alpha=.79$ and.72) and test-retest reliability ( $r=.68$ and.94) for the alienation and stereotype endorsement subscales has been reported in a previous study (18).

The Beck Hopelessness Scale (BHS) is a 20-item questionnaire that asks participants to endorse statements as true or false as applied to them (19). Individual items are then summed to provide an overall index of hope or its absence. This scale has been used successfully with a wide range of psychiatric, medical, and community populations.

The Rosenberg Self Esteem Schedule (RSES) was used to measure self-esteem (20). It is a tenitem self-report questionnaire that has been successfully used in a number of studies with persons with schizophrenia (9). Individual items are summed such that higher scores indicate higher self-esteem. 
The Ways of Coping Questionnaire (WCQ) is a self-report instrument that asks participants to recall a recent stressor and then rate how often they used any of 66 behaviors to cope with that particular stressor (21). The authors of this scale reported internal consistency scores in the range of .61-.76. Construct validity was supported by correlations with theoretically related constructs such as problem solving and locus of control. However, although this instrument has been established as a measure of coping in the general population, the factor structure of the scale, as with other scales, may not accurately reflect coping behaviors used by individuals with severe mental illness (22). In this study, we therefore developed, using two different samples, a rational scoring system that would be sensitive to coping deficits particular to severe mental illness (23). This scoring scheme yields six modes of coping scores, of which two were used in these analyses (these specific modes were selected because they most closely resembled the construct of avoidant coping): ignoring, which refers to putting the stressor out of one's mind or choosing to not think about it, and resigning, which refers to a choice to not act because the person perceives that there is nothing to be done. In one study that compared results derived from the original scoring system with our revised scoring scheme across two previous samples, the rationally devised scales had better internal consistency. Several of the original scale scores but none of the new scale scores failed to achieve acceptable internal consistency. In calculating scores, we used relative scores, as we have elsewhere (23). These are obtained for each scale by dividing the mean score for that scale by the mean score for the total test. This has the advantage of pointing to a participant's relative preference and corrects somewhat for response bias.

\section{Analyses}

Steps before path analysis-Several steps were taken before the path analysis in order to minimize the number of variables in the analysis so that power could be maximized. Hope and self-esteem (which we had already regarded to be conceptually linked) were found to be moderately correlated $(r=.63)$. Because of their high correlation and conceptual link, we decided to convert them to $\mathrm{z}$ scores and combine them into one scale. Additional scale combinations were based on conceptual linkages between the construct. As previously noted, two conceptually linked sub-scales of the WCQ (ignoring and resigning) were also combined into one scale, as were two conceptually linked subscales of the ISMIS (alienation and stereotype endorsement).

We then tested for moderation of insight and stigma by creating a product-term variable of centered versions of the SUMD and ISMIS variables. Initial regression analyses did not support that this variable significantly predicted hope and self-esteem when we controlled for ISMIS and SUMD, however. Thus the IS-MIS and SUMD were included in the path analysis as separate variables.

Path analyses-Before conducting the path analysis, we examined correlations between all variables to be included in the path models and other demographic variables. An initial path analysis was then conducted with AMOS 4.0 to test the theoretical model (employment was excluded, as was the interaction between internalized stigma and awareness). Next, drawing from Fialko and colleagues (13), we addressed in an alternative model the perspective that low self-esteem and suicidality result from distress about psychotic symptoms. On the basis of this perspective, positive symptoms were made an exogenous variable, along with stigma and SUMD, and relationships between positive symptoms and all the other variables were tested.

\section{Results}

A correlation-covariance matrix of all the variables included in the path analysis is presented in Table 1. As shown in Table 1, awareness (as measured with the SUMD) was significantly 
correlated with the PANSS depression item only, whereas internalized stigma was associated with all variables in the model except PANSS depression and SUMD awareness. The variable for hope and self-esteem was significantly negatively associated with all variables expect SUMD awareness, whereas avoidant coping was significantly associated with all variables except awareness and positive symptoms.

Additional correlations between demographic variables and variables included in the path analysis were explored. The only variables found to be related to the exogenous variables (internalized stigma and awareness) were, respectively, age at first hospitalization ( $\mathrm{r}=-.23, \mathrm{p}<$. $05)$ and lifetime number of hospitalizations $(\mathrm{r}=-.23, \mathrm{p}<.05)$. Including these variables as control variables in the path analyses did not alter findings in any meaningful way, so they were excluded to increase the power of the analyses.

Results of the first path analysis for testing our original model are presented in Figure 2. Model fit indices are presented in Table 2. Results supported the view that internalized stigma negatively influences avoidant coping, active social avoidance, and depressive symptoms. The results also show that this relationship was mediated by the impact of internalized stigma on hope and self-esteem. There was also evidence that internalized stigma affected positive symptom severity by way of its impact on social avoidance, but the predicted relationship between avoidant coping and symptom severity was not supported. Similarly, there was no support for an effect of avoidant coping on social avoidance. The lack of significant findings with regard to avoidant coping and social avoidance is interesting, given that there was a significant bivariate relationship between the two variables (as Table 1 shows). Thus the lack of a relationship suggests that the inclusion in the model of the more powerful variable of hope and self-esteem accounted for the impact of avoidant coping. There was no evidence of a significant impact of awareness on hope and self-esteem or on avoidant coping, although there was a nonsignificant trend for a relationship between awareness and depression. Model fit indices were good, suggesting that this model fit the data well.

Results of the second path analysis, which tested a modified model, are presented in Figure 3. The main difference between the two models is that the first model treats positive symptoms as an outcome whereas the second treats it as an input, or predictor of outcome. Model fit indices are presented in Table 2. All significant relationships between internalized stigma and the other variables supported in model 1 were replicated in this model. In addition, however, results supported the possibility that positive symptoms significantly influence hope and selfesteem, avoidant coping, and social avoidance. Model fit indices suggest that the alternative model also fit the data well.

\section{Discussion}

Findings from two path models provided support for the hypothesis that internalized stigma reduces a person's hope and self-esteem, leading to negative outcomes related to recovery, including depressive symptoms, social avoidance, and a preference for using avoidant coping strategies. Simply put, findings supported the hypothesis that the process of accepting and internalizing social stigma changes the way people perceive and feel about themselves and their likelihood to plan and meet their life goals and consequently leads them to avoid others and experience depression.

Even though our original model analysis provided support for the view that social avoidance is linked to low self-esteem and diminished hope and that these together lead to increased severity of positive symptoms, the alternate model we tested provided evidence that positive symptoms may also plausibly be seen as a cause of social avoidance and avoidant coping. Notably, however, changing positive symptoms from an outcome to a predictor did not have 
a meaningful impact on the relationship between internalized stigma (as mediated by hope and self-esteem) and the other outcome variables.

Some aspects of our original model were not supported. Our analyses did not support the finding from our previous study (7) that internalized stigma moderates the effects of awareness of mental illness on hope and self-esteem. The study reported here used a different method of assessing moderation (product-term analysis, as opposed to cluster analysis followed by a comparison of means), as well as a different measure of insight. It is possible that a restriction of range in insight, possibly related to how the SUMD measures awareness, may explain the failure to replicate the finding of moderation in these analyses.

In addition, although this study found evidence of a bivariate relationship between avoidant coping and social isolation, this relationship was not supported in the path analyses. Hope and self-esteem appeared to account for the observed relationship between these two variables. This finding may suggest that the links between coping and social isolation were largely a function of their mutual links with persons' expectations of the future and their appraisals of their personal value. As with all unexpected findings, further research is needed to clarify this issue.

There were several limitations to the study that are important to consider. Given the crosssectional nature of this study, we cannot draw definitive conclusions regarding causality, and alternative explanations of the findings cannot be ruled out. Notably, all participants were enrolled in vocational rehabilitation and had equivalent employment status, so we were unable to test the hypothesized impact of internalized stigma on employment status, an important construct in our original model. In addition, we did not have a variable representing suicidal ideation, so we used a depression item that may not have accurately represented this construct. Furthermore, participants were mostly men in their 40s, all of whom were involved in treatment. It may well be that a different relationship exists between the variables we measured among younger persons with schizophrenia, in a predominantly female sample, or in particular among persons who decline treatment. Thus more research is necessary and should involve the collection of data at multiple time points and with broader samples.

\section{Conclusions}

As we have discussed, we believe that the accumulating evidence supports that internalized stigma is negatively correlated with recovery-related outcomes, supporting the need for a standardized, replicable intervention to target internalized stigma. Integrating promising new cognitive-behavioral (24) and narrative enhancement approaches (25) may help consumers with severe mental illness to overcome internalized stigma and develop more hopeful attitudes and expectations about the future, thereby facilitating recovery. Cognitive-behavioral approaches may help individuals by addressing self-stigmatizing views that lead to hopelessness and low self-esteem. These views can be treated as cognitive distortions or dysfunctional attitudes that can be changed through the process of cognitive restructuring (collecting evidence to test and challenge the validity of beliefs). Narrative enhancement approaches may also be useful in addressing self-stigmatizing views that have been incorporated into one's life story. The end goal of narrative enhancement would be to help clients to tell stories about their lives in which their role as a protagonist is developed and transformed and themes of empowerment and agency (rather than hopelessness and powerlessness) are emphasized. These interventions may help to interrupt the process by which accepting and internalizing stigma leads to hopelessness and social avoidance, as our findings supported. 


\section{Acknowledgements}

This work was supported by National Institute of Mental Health grant R34-MH082161 to Dr. Yanos and by a Veterans Affairs Rehabilitation and Development grant to Dr. Lysaker.

\section{References}

1. Corrigan, PW.; Ralph, RO. Introduction: recovery as consumer vision and research paradigm, in Recovery in Mental Illness: Broadening Our Understanding of Wellness. Ralph, RO.; Corrigan, PW., editors. Washington, DC: American Psychological Association; 2005.

2. Liberman RP, Kopelowicz A. Recovery from schizophrenia: a concept in search of research. Psychiatric Services 2005;56:735-742. [PubMed: 15939952]

3. Resnick SG, Fontana A, Lehman AF. An empirical conceptualization of the recovery orientation. Schizophrenia Research 2005;75:119-128. [PubMed: 15820330]

4. Estroff SE. Self, identity, and subjective experiences of schizophrenia: in search of the subject. Schizophrenia Bulletin 1989;15:189-196. [PubMed: 2665052]

5. Roe D. Progressing from patienthood to personhood across the multidimensional outcomes in schizophrenia and related disorders. Journal of Nervous and Mental Disease 2001;189:691-699. [PubMed: 11708670]

6. Davidson, L. Living Outside Mental Illness: Qualitative Studies of Recovery in Schizophrenia. New York, New York: University Press; 2003.

7. Lysaker PH, Roe D, Yanos PT. Toward understanding the insight paradox: internalized stigma moderates the association between insight and social functioning, hope and self-esteem among people with schizophrenia spectrum disorders. Schizophrenia Bulletin 2007;33:192-199. [PubMed: 16894025]

8. Lysaker PH, Davis LW, Warman DM, et al. Stigma, social function and symptoms in schizophrenia and schizoaffective disorder: association across 6 months. Psychiatry Research 2007;149:89-95. [PubMed: 17156853]

9. Corrigan PW, Watson AC, Barr L. The self-stigma of mental illness: implications for self-esteem and self-efficacy. Journal of Social and Clinical Psychology 2006;25:875-884.

10. Ritsher JB, Phelan JC. Internalized stigma predicts erosion of morale among psychiatric outpatients. Psychiatry Research 2004;129:257-265. [PubMed: 15661319]

11. McCay EA, Seeman MV. A scale to measure the impact of a schizophrenic illness on an individual's self-concept. Archives of Psychiatric Nursing 1998;12:41-49. [PubMed: 9489173]

12. Bond GR, Resnick SG, Drake R, et al. Does competitive employment improve nonvocational outcomes for people with severe mental illness? Journal of Consulting and Clinical Psychology 2001;69:489-501. [PubMed: 11495178]

13. Fialko L, Freeman D, Bebbington PE, et al. Understanding suicidal ideation in psychosis: findings from the Psychological Prevention of Relapse in Psychosis (PRP) trial. Acta Psychiatrica Scandinavica 2006;114:177-186. [PubMed: 16889588]

14. Spitzer, R.; Williams, J.; Gibbon, M., et al. Structured Clinical Interview for DSM-IV. New York: Biometrics Research; 1994.

15. Kay SR, Fiszbein A, Opler LA. The Positive and Negative Syndrome Scale (PANSS) for schizophrenia. Schizophrenia Bulletin 1987;13:261-276. [PubMed: 3616518]

16. Bell MD, Lysaker PH, Beam-Goulet JL, et al. Five-component model of schizophrenia: assessing the factorial invariance of the Positive and Negative Syndrome Scale. Psychiatry Research 1994;52:295303. [PubMed: 7991723]

17. Amador XF, Strauss DH, Yale SA, et al. Awareness of illness in schizophrenia. Schizophrenia Bulletin 1991;17:113-132. [PubMed: 2047782]

18. Ritsher JB, Otilingam PG, Grajales M. Internalized stigma of mental illness: psycho-metric properties of a new measure. Psychiatry Research 2003;121:31-49. [PubMed: 14572622]

19. Beck AT, Weissman A, Lester D, et al. The measurement of pessimism: the Hopelessness Scale. Journal of Consulting and Clinical Psychology 1974;42:861-865. [PubMed: 4436473] 
20. Rosenberg, M. Society and the Adolescent Self-Image. Princeton, NJ: Princeton University Press; 1965.

21. Lazarus, RS.; Folkman, S. Stress, Appraisal and Coping. New York: Springer; 1984.

22. Wineman NM, Durand EJ, McCulloch BJ. Examination of the factor structure of the Ways of Coping Questionnaire with clinical populations. Nursing Research 1994;43:268-273. [PubMed: 7937172]

23. Lysaker PH, Johnannesen JK, Lancaster RS, et al. A rationally devised scoring scheme to assess coping in schizophrenia: internal consistency and associations with work performance. International Journal of Psychosocial Rehabilitation 2004;8:41-51.

24. Knight MTD, Wykes T, Hayward P. Group treatment of perceived stigma and self-esteem in schizophrenia: a waiting list trial of efficacy. Behavioural and Cognitive Psychotherapy 2006;34:305-318.

25. Lysaker PH, Buck KD, Roe D. Psychotherapy and recovery in schizophrenia: a proposal of key elements for an integrative psychotherapy attuned to narrative in schizophrenia. Psychological Services 2007;4:28-37. 


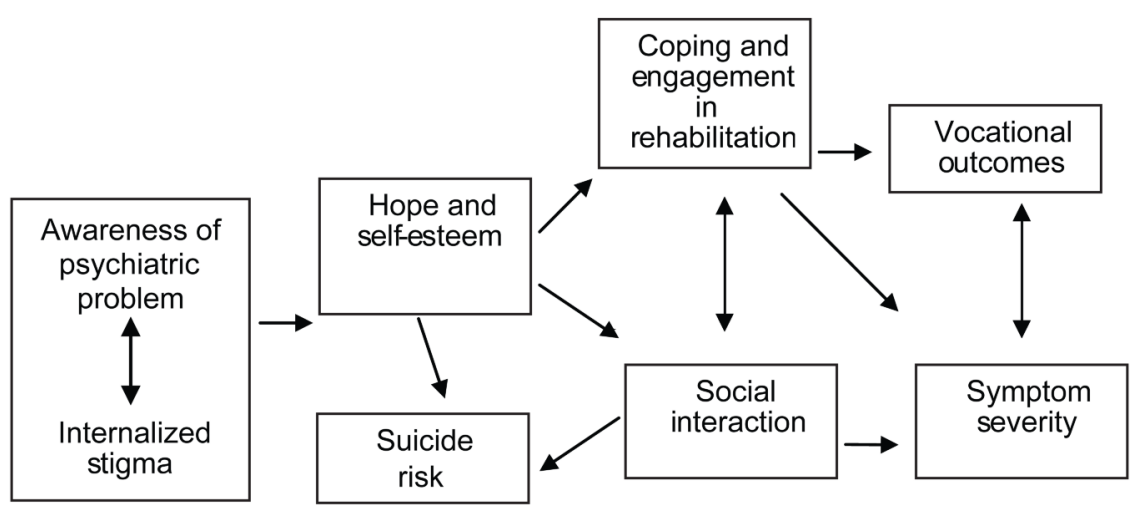

Figure 1.

Model of the hypothesized impact of internalized stigma on recovery-related outcomes for persons with severe mental illness 


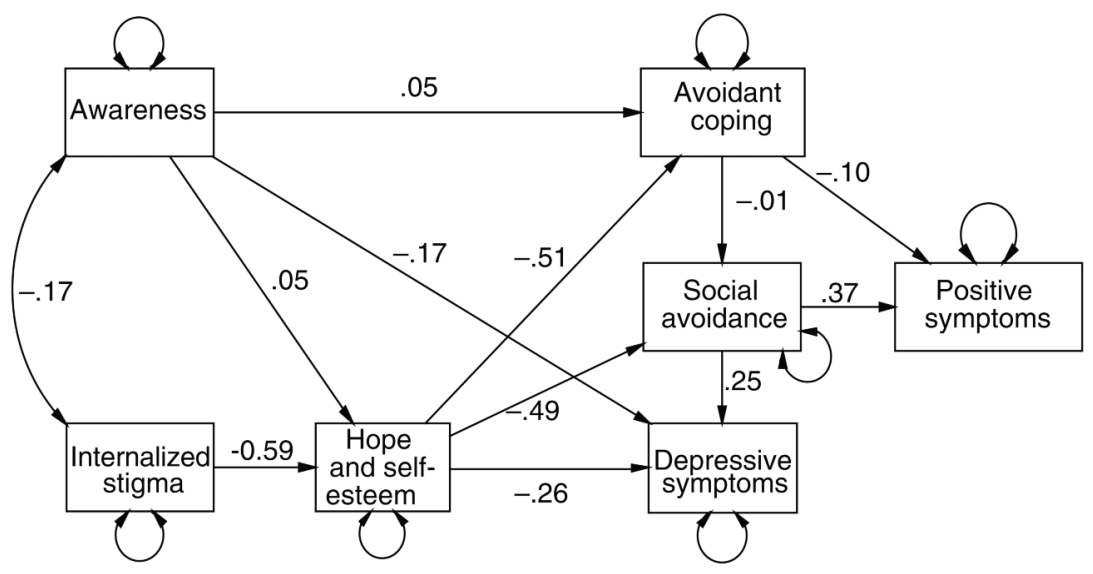

Figure 2. Path model 1, where positive symptoms of schizophrenia are treated as an outcome $\mathrm{e}^{\mathrm{a}}$ a $\mathrm{N}=102$. Standardized coefficients are presented. 


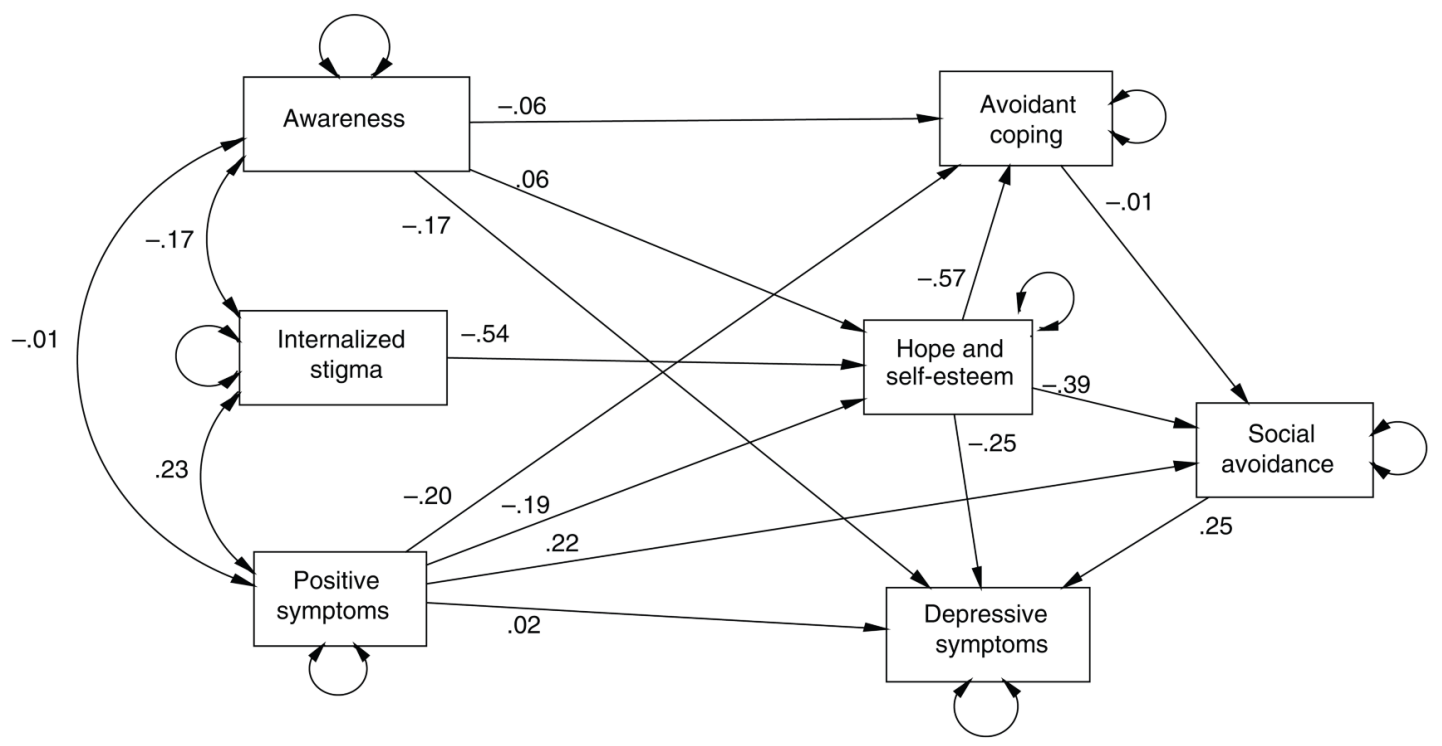

Figure 3. Path model 2, where positive symptoms of schizophrenia are treated as input ${ }^{\text {a }}$ ${ }^{a} \mathrm{~N}=102$. Standardized coefficients are presented. 


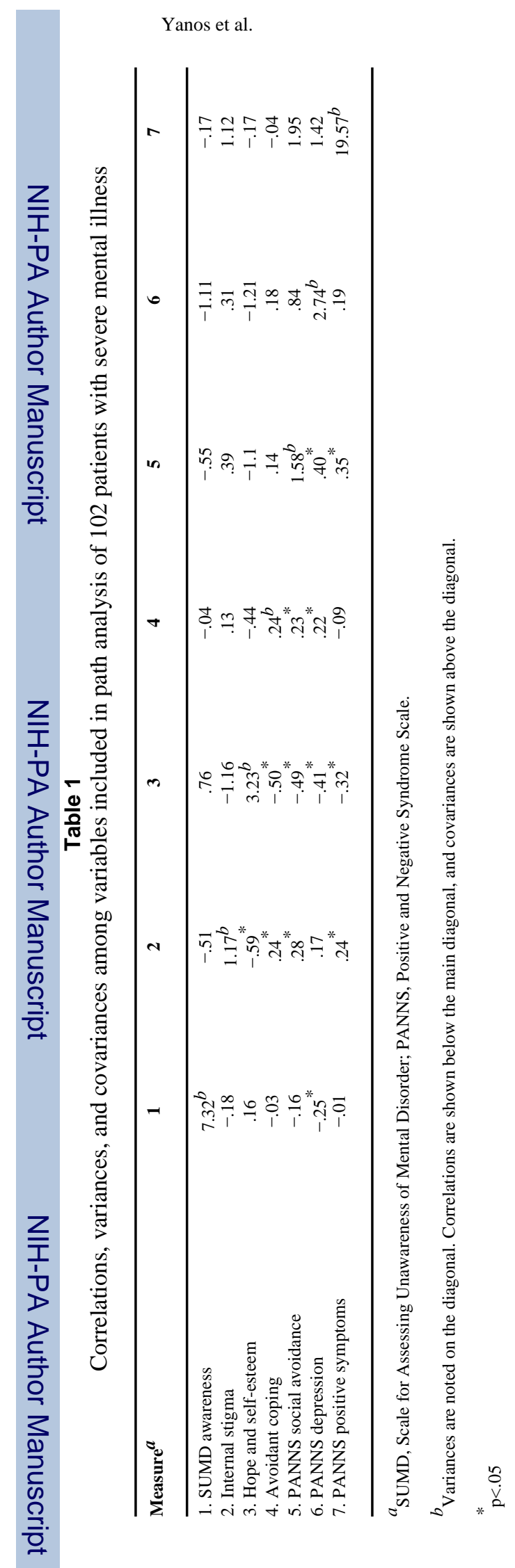

Page 12 
Table 2

Model fit indices for path models of internalized stigma with schizophrenia

\begin{tabular}{|c|c|c|}
\hline Measure & Model 1 & Model 2 \\
\hline $\begin{array}{l}\chi^{2} \\
\text { Df } \\
\text { P } \\
\text { Comparative fit index } \\
\text { Adjusted goodness-of-fit index } \\
\text { Economic vulnerability index } \\
\text { 95\% CI } \\
\text { Root mean square error of association } \\
\text { 95\% CI }\end{array}$ & $\begin{array}{c}11.59 \\
9 \\
.23 \\
.98 \\
.90 \\
.49 \\
.47-.62 \\
.05 \\
0-.13\end{array}$ & $\begin{array}{c}11.34 \\
7 \\
.12 \\
.97 \\
.88 \\
.53 \\
.49-.66 \\
.08 \\
0-.16\end{array}$ \\
\hline
\end{tabular}

